\title{
Jacques Taxi
}

Le Professeur Jacques Taxi est décédé le 5 décembre à l'âge de 95 ans.

Issu d'une famille modeste, il poursuit ses études secondaires comme boursier et passe le baccalauréat en juillet 1940. En octobre, il entre en SPCN à la Faculté des Sciences de Paris, devenue après 1968 l'UPMC, qu'il ne quittera plus, sauf en 43-44, car réfractaire au STO puis résistant, il doit alors se réfugier dans le Cher, à Boulleret, village d'origine de sa mère, où il passait toutes ses vacances et où il vient d'être inhumé.

Après avoir achevé sa licence et préparé un DES, il obtient en 1949 l'agrégation de Sciences Naturelles mais, dès 1947, M. Couteaux lui avait proposé un poste d'assistant dans son service. C'était le début d'une très longue carrière d'enseignant chercheur qui, de la rue Cuvier au quai Saint-Bernard, allait mener Jacques Taxi bien au-delà de sa retraite. En effet, lorsque je lui succédai en 1988 à la tête du laboratoire de Cytologie intégré à l'Institut des Neurosciences, il poursuivit pendant près de 30 ans, comme professeur émérite puis collaborateur bénévole, une activité de recherche au service des équipes du laboratoire qui avaient besoin de données cytologiques. La dernière de ses publications est de 2017 : avec l'équipe de Fatiha Nothias, J. Taxi y explorait l'ultrastucture de la moelle épinière réparée après traumatisme. Auparavant, dans un travail paru en 2014, les mêmes chercheurs lui avaient permis de retrouver le système nerveux périphérique.

De fait M. Couteaux, pour qui il devait conserver toute sa vie un respect filial et une admiration émerveillée, lui avait confié comme sujet de thèse l'étude de l'innervation $\mathrm{du}$ muscle lisse intestinal et c'est sur le système nerveux végétatif (SNV), notamment les ganglions sympathiques que Jacques Taxi allait acquérir une notoriété durable. La mise en évidence de la libération non-synaptique des médiateurs dans le SNV ouvrait notamment la voie vers d'autres modes de communication nerveuse que les articulations synaptiques. Pour ces travaux, encore non dépassés à ce jour, J. Taxi avait eu le mérite de mettre en œuvre, après les méthodes tinctoriales puis histochimiques, la microscopie électronique. Avec cet instrument, qu'il manipulait encore à 93 ans avec autant de joie que d'efficacité, il pouvait en effet mettre en évidence les différents éléments structuraux impliqués dans la libération et l'action des médiateurs et notamment les «Corps de Taxi», structures post-synaptiques au rôle encore énigmatique. Son éclectisme dans le choix des modèles animaux les plus adaptés au problème étudié allait le mener de la souris à la grenouille ou au poisson zèbre chez lequel il étudiait encore récemment avec Caroline Parmentier le système neurosécrétoire caudal. Ainsi, parmi les mots-clés de sa recherche sur la neurotransmission, les neuropeptides font suite à la noradrénaline et à l'acétylcholine tandis que, pour les techniques, l'immunocytochimie s'est ajoutée à la radioautographie, mise en œuvre avec Bernard Droz, tandis que Daniel Eugène lui apportait l'électrophysiologie.

Mais Jacques Taxi était aussi un enseignant respecté et apprécié : du PCEM au DEA de Cytologie qui a formé dans son laboratoire des générations d'histologistes français, il a couvert tous les niveaux d'enseignement universitaire et il a continué après sa retraite à être une référence et un conseiller ouvert et accessible pour les étudiants, jeunes chercheurs et stagiaires de l'IDN. Parmi ces derniers, il faut souligner la présence en nombre de ceux issus des pays de l'Est, notamment la Russie dont Jacques Taxi parlait la langue et avec qui il avait noué, dès l'époque soviétique, des liens solides que j'ai continué à développer, en particulier avec le laboratoire de l'Académie des Sciences dirigé par Mikhaïl Ugrumov à Moscou.

Mikhaïl m'écrit qu'avec M. Taxi il perd un modèle d'humanisme, un mentor et comme un père spirituel. De fait les qualités humaines de Jacques Taxi, sa disponibilité, sa modestie, sa bienveillance, qui n'empêchaient pas l'autorité du «patron», rendaient sa personnalité particulièrement attachante. Pendant les vingt ans d'une fréquentation presque quotidienne, j'ai pu apprécier comment il avait su d'emblée trouver sa place d'émérite sans rien revendiquer mais dans le bonheur constant d'être enfin à temps plein «chercheur de base », attaché autant à son microscope qu'à une blouse qui ne le quittait jamais... Cette équanimité permanente était due aussi à la stabilité et à la générosité d'un foyer, riche de 70 ans de vie commune et où, depuis plus de 30 ans, son épouse et lui ont accueilli et entièrement élevé leur petit-fils, gravement handicapé. 
Éloigné par un agnosticisme congénital de toute transcendance, Jacques Taxi regrettait cependant la disparition des références chrétiennes (mais s'effarait de leur retour en Russie..). Pour lui, les valeurs fondamentales étaient celles du chercheur : travail, probité, gratuité et, par-dessus tout, liberté. Loin de tout carriérisme et, comme il le disait, des préoccupations du «management », il a pu et su les mettre en œuvre au sein de cette structure ingérable mais si accueillante qu'est notre université.
Après Mme Fessard, les Buser, Laget, Galifret... disparaît avec Jacques Taxi une génération glorieuse de neurobiologistes de l'UPMC qui, par leur travail et leurs capacités de synthèse ont su non seulement concilier mais associer harmonieusement l'enseignement et la recherche, les deux poumons de l'Université.

Bordeaux, le 21 décembre 2017

André Calas 